\title{
Simon Gantillon et l'expressionnisme dramatique
}

\author{
Simon Gantillon and dramatic expressionism
}

Tomasz KACzmareK [tkn@wp.pl]

Uniwersytet Łódzki, Pologne

\begin{abstract}
RÉSUMÉ :
Peu nombreux sont ceux qui s'intéressent aux problèmes de l'esthétique expressionniste dans la dramaturgie française. Sans aucun doute, cette lacune est à mettre au compte d'une méconnaissance de ce mouvement en France. Certainement, le pays de Voltaire n'a pas donné de textes comparables à ceux qu’on rencontre dans le mouvement allemand, mais il est évident que parmi les dramaturges français on pourrait trouver des auteurs dont les œuvres ont été conçues selon cette conception artistique. Simon Gantillon appartient à ces écrivains qui, sous l'influence d'August Strindberg, ont cherché une nouvelle expression, étrangère au «cartésianisme » régnant sans partage depuis des siècles sur les tréteaux. L’auteur français n'est pas un expressionniste, mais il a adopté une nouvelle formule, surtout en ce qui concerne la construction des personnages, qui le rapproche des avant-gardistes d'outre-Rhin. C'est dans cette perspective que dans le présent article on analyse les trois pièces du dramaturge dans lesquelles nous assistons au dédoublement du protagoniste (Maya), à l'étude des âmes inquiètes face au climat néfaste (Cyclone) et à la fuite devant la grisaille de la vie quotidienne (Départs). De fait, Gantillon présente dans ses drames sa vision personnelle de l'art nouveau qui lui a garanti un succès incontestable de son vivant.
\end{abstract}

\section{MOTS CLÉS :}

Simon Gantillon ; Gaston Baty ; expressionnisme ; féerie ; dédoublement de la personnalité

\begin{abstract}
:
Not many scientists are interested in the issues related to the expressionists' esthetic in the French theater. For sure this is caused by the common opinions that this vanguard movement was not represented in France. It is obvious that the Volaire's homeland did not produce piece works comparable to these present in German movement, despite this fact, some French writers were influenced by the German artistic concepts. One of them was Simon Gantillon who being under the influence of August Strinberg searched for the new means of expression strange for the Cartesian taste dominating in Parisian and worldwide theaters. The French author is not the expressionist writer par excellance, but in his works the German heritage could be seen especially in the construction of the characters. Therefore in this perspective, in the article the analysis of the three Gantillon's plays is presented where the readers can participate either in the schizophrenia of the protagonist (Maya), or study of non-humble souls confronted with the morderous climate (Cyclone) or in the escape from the routine of every-day life (Départs). Gantillon presents in his dramas his own vision of the new art which immortalized him during his lifetime.
\end{abstract}

\section{KEYWORDS:}

Simon Gantillon; Gaston Baty; expressionism; féerie; schizophrenia 


\section{Introduction}

Il existe des dramaturges qui créent dans la solitude et dont les œuvres exercent une fascination sur la postérité, et il y en a d'autres qui, après les succès remportés de leur vivant, tombent dans l'oubli et leur étoile s'éclipse. C'est le cas de Simon Gantillon (1887-1961) qui, tout de même, jouissait d'une renommée dramaturgique incontestable. Sa gloire remonte à l'entre-deux-guerres, quand cet ancien navigateur collaborait étroitement avec un génie en matière de mise en scène française : Gaston Baty. Il serait intéressant d'essayer de comprendre comment et pourquoi un dramaturge de cette envergure a si vite disparu des scènes. On écrit encore sur lui dans différents manuels dédiés au théâtre ou à l'histoire de la littérature, mais on lui réserve uniquement quelques mots plus que liminaires (Cf. Surer 1964 : 44 ; Simon 1970 : 152).

Formé à l'école réaliste, Gantillon ne lui restera pas longtemps fidèle. Du moment où la réalité extérieure a cessé de lui procurer les sujets, il décide d’étudier les obscurités du psychisme humain. C'est ainsi qu'il tourne le dos à la tradition typiquement française pour chercher d'autres moyens d'expression. De fait, l'écrivain ne saurait être désigné comme un auteur réaliste, encore moins naturaliste puisque la constitution psychique de ses personnages échappe à toute vérification logique. Il n'est pas non plus un auteur symboliste pour autant, car la symbolique des objets ou des situations qu'il adopte dans certains de ses drames ne reflète que les états d'âme des protagonistes et sert de fond seulement à l'action que l'on pourrait qualifier de psychanalytique. C'est à ce propos qu'en étudiant les pièces de Gantillon, nous nous sommes rendu compte que leur contenu reste en relation avec l'expressionnisme. Cette œuvre hétéroclite retrouve en effet sa cohérence dès qu'on l'analyse dans le contexte de l'expressionnisme dramatique. Cette approche est d'autant plus légitime que l'écrivain a souvent recours à la " féerie expressionniste » qui « débouche sur l'ambiguité, l'ambivalence, l'amour-haine, l'angoisse, elle fait de nous des étrangers dans le monde»(Gravier 1971 : 294).

\section{Cyclone, ou l'évasion du réel}

Gantillon débute en tant que dramaturge avec Cyclone qui sera monté à la Baraque de la Chimère le 29 mai 1923 dans les décors de Georges Rouquayrol. La pièce reprend le motif des premières expériences maritimes de l'écrivain qui a passé beaucoup de temps en pleine mer. «On dirait que l'auteur a vécu lui-même de longs mois dans le poste d'équipage d'un voilier pour arriver à reconstituer aussi fidèlement les gestes et les propos échangés entre matelots » (Villeroy 1923: 212). Le dramaturge ne cache pas qu'il s'inspire de ses propres expériences quand il décrit les dangers que la mer réserve aux chercheurs d'aventure. Néanmoins, ses descriptions pittoresques, qui rappellent parfois la narration de Pierre Loti, servent de toile de fond pour étaler la décomposition psychique des marins sous l'influence d'un climat destructeur. Gantillon brosse le portrait des individus face à une catastrophe imminente, le cyclone étant un élément hautement dramatique qui contribue à la désagrégation intérieure de l'âme humaine.

C'est ainsi que l'écrivain français situe l'action du drame dans le poste d'équipage d'un voilier marchand tout en respectant les détails inhérents au travail des marins. Tout porterait à croire que Gantillon veut respecter rigoureusement les contraintes naturalistes. Et pourtant, 
cette pièce contient les particularités discrètes d'une esthétique qui donne un démenti à l'art foncièrement réaliste. Marcel Raymond déclare que le dramaturge «s'affranchit de la copie de la vie pour atteindre le réalisme théâtral » (Raymond 1943: 86), mais cette approche vise à la « spiritualisation de la partie matérielle et à la matérialisation de la partie immatérielle » (Sarrazac \& Marcerou 1999 : 23). De fait, l'auteur procède graduellement pour créer une atmosphère de plus en plus spirituelle, en accord avec une «vision thomiste " (Tariol $1980: 15$ ) lancée par Baty. Afin de percer la surface du visible, il songe à exprimer l'équilibre entre " l'esprit et la matière, l'homme et les choses » (Simon $1972: 148$ ).

Dès le premier dialogue, le dramaturge anticipe l'étrangeté qui s'installera dans les cœurs des marins. Tout d'abord, l'approche du cyclone et de la chaleur insupportable détraquent leur psychisme. Les matelots crient, tels des protagonistes expressionnistes, comme s'ils étaient dans une chaudière infernale : "qu’on sorte de cette poêle à frire ", " on se brûle aux rambardes des passerelles », « l'électricité me sort par les doigts de pied ». Les effets sonores déstabilisent aussi les pauvres abandonnés en pleine mer au même point que le climat étouffant:

LE BORDELAIS : Il commence - à me taper sur le système avec sa musique d'enterrement... Voilà une heure que ça dure...

LE PARIGOT : Si seulement il faisait pleuvoir...

AGUISTACE : Pense à la mort, ça aide à vivre...

LE BORDELAIS : Du moment qu'on te sert du vague à l'âme !... Moi, ces airs-là, ça me rebrousse les nerfs... J'ai envie de grincer des dents... ou de mordre... (Gantillon 1923 : 2).

À ce stade de la lecture on pourrait croire que Gantillon étale devant nous une bande de voyageurs qui tout simplement ne résistent pas au climat insoutenable, mais au cours de l'action nous voyons comment ce climat évoque l'état d'âme des protagonistes. Le cyclone, qui est incontestablement un phénomène bel et bien réel, symbolise également une force étrangère à ce monde et génératrice d'un sens figuré propre à créer une ambiance irréelle. Le cyclone personnifie donc les remous du subconscient quéveillent les vents impétueux ; plus encore, il constitue l'annonce d'une réalité invisible que les symbolistes et les expressionnistes voulaient susciter dans leurs drames. Il n'est pas étonnant que Le Novice, sous l'emprise de la bourrasque, se prononce sur l'existence d'un univers parallèle :

Vous ne croyez pas qu'il y a des choses qu'on ne sait pas... que personne ne sait... que personne ne saura jamais ?... des choses, comme ça... qu'on ne voit pas... qu'on n’entend pas... qu'on n'explique pas... mais qui sont quand même autour de nous... auxquelles on en peut pas échapper ?... Aguistace m'a dit que c'est la lune qui fait monter et descendre les marées... Les hommes sont moins forts que la mer... Eux aussi, peut-être qu'ils vont, qu'ils viennent parce qu'ils sont commandés par une... une espèce de... Enfin (Gantillon 1923 : 12).

Dès lors, nous assistons à la diminution psychique des personnages qui se meuvent comme dans un rêve. Tout est enveloppé de mystère. Le quart de Scavine dans lequel Aguistace a bu constitue le moment crucial de la métamorphose du personnage. Hypnotisé, il sera persécuté par l'ombre du Russe (que quelqu'un a poussé dans la mer), qui selon lui, le blessera à la tête. De 
plus, une femme étrangère qui rappelle une sirène incite le marin magnétisé à l'attraper dans les profondeurs de la mer. Au point culminant de la pièce qui précède la fin d'Aguistace (il se jettera dans l'eau), le monde extérieur cède la place à l'inconnaissable et au ténébreux. C’est de cette manière que Gantillon veut center l'action sur l'évasion, motif privilégié des expressionnistes. Tout finit par le chant des matelots, censé les encourager, qui se perd au loin. Tout se dilue dans le fond de la mer et voici que la scène finale touche à sa fin.

Malgré les longs dialogues, cette œuvre ne s'avère qu'un drame statique qui relate les errements psychologiques des protagonistes. Lécrivain table sur les déchirements que subissent leurs âmes en proie à la douleur existentielle et ces souffrances acquièrent encore plus d'importance dès qu'on les analyse depuis la perspective expressionniste.

\section{Maya, ou le triomphe de l'illusion}

Un an après la parution de Cyclone, Gantillon travaille sur un nouveau drame qui lui garantira un succès international, Maya, traduit en seize langues. L'histoire d'une femme de mœurs légères fascine aussitôt la capitale française. «C’est un poème à la gloire de la prostituée, dispensatrice des douces joyes aux hommes qui s'ennuient : exilés, marins, colons » (Raymond 1943 : 86). Les spectateurs arrivent en grand nombre pour voir la maison de passe dans laquelle des filles de tous âges s'adonnent délibérément au trafic de la chair. Cependant, les avides d'histoires égrillardes quitteront le théâtre déçus. Si on voulait se limiter à son aspect purement extérieur, il serait opportun de constater que l'intrigue relate tout simplement la vie des prostituées comme dans un feuilleton. Néanmoins, comme dans sa première pièce, le dramaturge n'a aucune intention d'écrire une pièce naturaliste. L'auteur renonce tant qu'il peut aux vulgarités et aux détails de la vie quotidienne afin d'accentuer la dimension poétique de l'existence des demi-mondaines. Dans le sillage du théâtre d'évasion, l'auteur campe ses pauvres créatures déchirées entre les désirs corporels et spirituels. En tant qu’observateur impassible, il ne porte pas de jugement, mais analyse les remous psychologiques, les disgrâces, qui ont touché ces femmes déchues. Gantillon ne présente donc pas des femmes ordinaires, sexuellement inapaisées et populacières, mais des personnes profondément malheureuses. Plus encore, ces amazones ne semblent pas être de ce monde matériel et, en particulier Bella, elles rappellent plus des êtres éthérés que des êtres en chair et en os, ce qui pourrait être déroutant compte tenu de leur profession.

Lécrivain attire notre attention surtout sur une prostituée, Bella, qui rappelle des personnages parfaitement expressionnistes. Or, Gantillon la présente comme un être qui se dédouble, procédé introduit par Strindberg et souvent utilisé par les Allemands. L'auteur note dans les indications scéniques qu' « il importe que le public ait, une impression visuelle immédiate, le sentiment que Fifine, Bella et la Vieille [femmes de mauvaise vie] sont la même fille à trois épisodes de sa vie »(Gantillon 1927 : 17). De là découlent deux constatations d'un intérêt considérable : d'abord, le primat que l'auteur donne à l'imaginaire au détriment du réel, et, ensuite, la décomposition psychologique de Bella. Elle sera représentée par deux personnes différentes qui la reflètent à diverses étapes de son existence. Gantillon joue ainsi avec le temps en superposant le passé et le futur, ce qui renforce l'effet de l'étrangeté des personnages. C’est par ce biais qu'il « cherche la vérité mystique dans la toute puissance de l'Amour 
et révèle, sous les apparences quotidiennes, d'inquiétants dédoublements » (Jomaron 1981 : 80). Ce procédé de dédoublement témoigne du vif intérêt que le dramaturge réserve à son héroïne et surtout à sa vie intérieure.

De fait, nous devons percevoir Bella non sous ses apparences physiques mais tenter de découvrir les méandres de son psychisme. Il n'est donc pas étonnant que Gantillon intitule sa pièce Maya en faisant ouvertement allusion à la philosophie hindoue. Loin d'aspirer à devenir un spécialiste de la sagesse orientale, il étudie le bouddhisme afin de créer son protagoniste. La prostituée symbolise Maya, car elle est la personnification de l'illusion. On dirait que dans ce drame il n'y a pas une intrigue classique et que l'écrivain, au cours de neuf tableaux, rythme l'évaporation progressive de Bella. Dans le dernier tableau, un barman hindou s'exprime sur la figure de la femme en lui attribuant un caractère énigmatique. Il insinue que derrière son apparence physique existe une autre créature, celle qui dupe nos sens :

LE GUITARISTE : (un temps) Elle s'appelle Bella, n'est-ce pas?

L'HINDOU : (soudain mystérieux) Aux Indes, son nom était Maya...

LE GUITARISTE : Maya... est-ce que ça veut dire quelque chose?

L'HINDOU : On ne sait pas bien.

LE GUITARISTE : C'est pas une araignée de mer, qui vit dans les herbes?

L'HINDOU : Quelquefois ça veut dire : "l'impossible à atteindre » et d'autres fois : Mère-dudésir... et : Sœur-du-Mensonge... ou bien, Maya, c'est l'apparence - tu sais... les choses... on croit qu'elles sont pas comme ça... et puis elles sont... autrement... Apparence... illusion... oui : puissance de l'illusion - surtout... (gestes professionnels) Illusion... illusion... illusion... Cock-tail-Maya !... Monsieur est servi !

LE GUITARISTE : Elle est belle?

L'HINDOU : Maya, ça veut dire aussi : »belle comme la description du livre » (Gantillon 1927 : 98).

C'est de cette manière que l'héroïne, conformément à l'art expressionniste, perd son humanité pour devenir l'incarnation d'une idée ; symbole puisant dans l'ancienne tradition. En effet, la philosophie spéculative védique présente Maya comme l'illusion du monde physique, celui-ci étant considéré par notre conscience comme la seule réalité existante. Les recherches spirituelles essayent de briser ce voile mensonger (l'illusion trompeuse) qui interdit d'apercevoir la vérité transcendante. Maya renvoie aussi aux concepts selon lesquels la réalité n’est pas plus tangible qu'un rêve.

Dans la tradition occidentale, l'illusion cacherait le " néant relatif » (Schopenhauer 1970 : 2), pour reprendre le terme de Schopenhauer, et c'est pourquoi Bella-Maya se montre tout d'abord comme la déesse qui possède le voile trompeur. Mais elle n'est guère cet esprit malin d'un succube. Les indications de Gantillon ne trompent pas. Le dramaturge dit clairement que Bella « est un personnage mythique, apparence et illusion, vendeuse de plaisir et pourvoyeuse de rêves, terrible et douce comme la nature ; Bella-Maya, femme et fée, élevée du signe particulier au symbole universel» (Gantillon 1927 : 5). À ce propos, il serait tentant de la comparer avec la figure de Maya dans Le Songe de Strindberg. Ce dernier précise ainsi les attributs du protagoniste de son drame : «Brahma, la force divine originelle, s'est laissé séduire par Maya, la mère du monde. Cette union entre la divine matière originelle et la matière terrestre provoqua 
la chute du ciel. C’est pourquoi le monde, la vie et les hommes ne sont plus qu'un fantôme, une apparence, un songe » (Strindberg $2006: 78$ ).

Pour les visiteurs du boxon, l'illusion (Maya) est devenue tout simplement réalité. Nous retrouvons la même approche dans l'œuvre de Strindberg qui, pour sa part, fait suite aux sortilèges de Maya, répandus dans les écrits d'Arthur Schopenhauer. Le philosophe allemand remarque à maintes reprises la figure de Maya en accentuant son rôle de trompeuse : " Le monde étend devant le regard de l'individu brut le voile de Maya, dont parlent les Hindous; ce qui se montre à lui, à la place de la chose en soi, c'est le phénomène seul, sous les conditions du temps et de l'espace » (Schopenhauer 1970 : 189). Nous observons alors la manière dont Bella entre progressivement dans la fonction d'une déesse qui au début réconforte ses clients pour, par la suite, personnifier la Maya indienne. Dans la scène avec Le Soutier elle se donne des airs de consolatrice:

Oui, mon gars, oui... raconte tes peines, puisque ça te fait du bien... j’y peux rien, bien sûr... Mais ça dégonfle [...] Pleure, si tu veux... Faut pas avoir honte... Devant moi !... Je vous connais, depuis que je vous pratique... je sais bien que vous crânez souvent, pour avoir l'air malin, à cause des copains... ou pour se donner du courage, de la patiente... hein ?... je sais bien... et que, dans le fond, vous êtes tout petits, tout petits... (Gantillon 1927 : 12).

La succession des tableaux juxtapose constamment le réalisme et le point de vue qui transperce la matérialité. Sous cet angle, la construction témoigne de la mise en valeur de l'imaginaire. Seule l'imagination transforme le réel et Gantillon agrémente son texte d'éléments visuels qui préparent le terrain au rituel. Tant s'en faut, puisque la nature est toujours opposée à l'artefact. Tels les expressionnistes qui préfèrent donner une vision plutôt qu'une illustration concrète du monde, Gantillon tourne le dos à toute objectivité au profit de l'expression d'une subjectivité passionnée. Ce procédé permettra à l'acteur de mieux étaler les remous de l'âme du personnage et au spectateur, comme dirait Felix Emmel, de faire « le plein usage de son œil intérieur et de son imagination »(Bablet $1971: 198$ ).

\section{Départs, ou la fuite devant soi-même}

Dans sa troisième pièce Départs (1928), le dramaturge semble reprendre le motif de la quête de l'homme cherchant à se libérer de la matérialité de la vie. En effet, dans la préface nous pouvons lire ceci : "l'homme souffre dans sa prison de chair, il tente d'en sortir, il échoue, il recommence » (Gantillon 1928b : 7). L’auteur de Maya préfère peindre donc, comme dans ses pièces précédentes, la collision entre notre spiritualité et notre corporéité, il désire « donner un tour dramatique au banal conflit de la vie et du rêve, et aussi - et surtout - aux vertus créatrices de l'imaginaire »(Gantillon 1928b : 7).

Gantillon brosse devant nous des rêves de jeunesse pleins de désirs souvent idéalistes qui seront cruellement confrontés à la réalité décevante. Il nous invite à participer à l'itinéraire imaginaire de deux jumeaux de quinze ans, Jeanne et Jean, qui vont extérioriser leurs phantasmes. Dès le premier tableau, l'auteur nous transporte dans le grenier de la maison de province où les protagonistes 
se composent en marge du quotidien un petit univers fiévreux et passionné. Dormeurs éveillés, possédés de l'éternel (et si moderne) besoin d' " autre chose » et d' " ailleurs », ils jouent à de magnifiques aventures, ils s'y laissent prendre parfois. Jeanne sera une actrice célèbre pour changer cent fois d'âme, toucher à toutes les gloires, connaître toutes les amours ; jean sera un grand navigateur, pour échapper à l'existence bourgeoise, découvrir l'inconnu, être libre enfin. Ah ! partir partir! (Gantillon 1928b : 7).

$\mathrm{Au}$ premier abord, il faut noter que la pièce ne se construit pas autour d'une intrigue bien encerclée. Nous assistons à des scènes qui se superposent comme dans les meilleurs drames américains des années trente, où les aventures des protagonistes se mélangent sans ambages à celles d'autres comparses. La première partie qui englobe les quatre tableaux annonce le début de l'ascension intérieure des personnages qui, enfermés dans le grenier comme s'ils se trouvaient sur un navire, s'imaginent un avenir radieux. Leur idéalisme sera fortement en contraste avec le prosaïsme des parents, figés dans le bourgeoisisme rétrograde et hostile à la jeunesse. Gantillon ne procède tout de même pas comme ses collègues allemands et ne concentre pas sa pièce sur le conflit entre générations, il en montre seulement le gouffre qui les sépare. Les jeunes ne se révoltent donc pas, à moins qu'on considère comme rebelle leur envie d'évasion dans un monde imaginaire. Mais c'est plutôt une résignation qu'une tentative de révolte.

Néanmoins, ce qui rappelle la méthode expressionniste, c'est la façon qu'a Gantillon de schématiser ses personnages au possible, surtout dans le tableau d'ouverture. Nous voyons les jeunes porter de grands masques chinois qui expriment deux sentiments différents : tragique et bouffon. Ils se déguisent et se livrent à un jeu verbal qui n'est pas sans rappeler les séances de psychodrames :

JEANNE : Ready.

JEAN : (lançant le mot) «Crépuscule »... [...]

JEANNE : Crépuscule... Forêt à l'automne...

JEAN : soleil pâle...

JEANNE : sur des futaies...

JEAN : futaies...

JEANNE : rousses...

JEAN : un cerf boit...

JEANNE : au bord d'un étang...

JEAN : ... d'un étang...

JEANNE : ... d'où monte une brume légère... (elle s'arrête)

JEAN : Heu ! Court... Court et chromo.

JEANNE : Un peu rouillée !... (elle fait tournoyer sa raquette)

JEAN : (même jeu) Play?

JEANNE : Ready.

JEAN : « Pirogue » (Gantillon 1928b : 16-17).

En s'aiguisant l'imagination par l'évocation verbale d'endroits exotiques, ils s'emballent à tel point qu'ils perdent leur propre identité. Ils sont bel et bien en transe extatique. Les deux 
protagonistes s'identifient à leurs personnages, ce qui ne les empêche pas de changer de sexe, tel Jean qui se transforme momentanément en Barbara, personnage indispensable dans l'histoire imaginée par Jeanne et sans laquelle elle ne pourrait guère poursuivre son jeu.

La deuxième partie du drame (tableaux 5 à 7) délaisse le registre poétique en mettant en scène le port à l'instant du chargement d'un navire, mais la troisième partie (tableaux 8 à 11) est entièrement concentrée sur la confrontation des désirs juvéniles face au désenchantement qui découle de la réalisation des rêves. Afin de souligner la désillusion, le dramaturge compare le mal des protagonistes avec celui des frères jumeaux en recourant à deux scènes parallèles. En regardant respectivement leurs photos, ils tiennent presque les mêmes propos, le procédé visant à représenter les protagonistes comme deux moitiés douloureusement éloignées. Le motif des répétitions de répliques atteint son paroxysme (l'auteur procède par phrases simples qui au moment culminant seront privées de verbe, pratique employée largement par les poètes expressionnistes) et rappelle plutôt un libretto qu'un dialogue dramatique. Gantillon semble payer sa dette envers Maurice Maeterlinck. La scène tourne en un récitatif qui reprend les mêmes mots, une sorte de fausse stichomythie :

Loge

JEANNE : Tu as bien choisi, toi...

Cabine

JEAN : Tu as bien choisi, toi...

Loge

JEANNE : La cabine, où l'on peut se recueillir...

Cabine

JEAN : La loge où l'on peut concentrer son ardeur...

Loge

JEANNE : La fraternité des coureurs de mer...

Cabine

JEAN : La franche camaraderie des artistes...

Loge

JEANNE : Lodeur puissante des vagues...

Cabine

JEAN : Les parfums grisants de la scène...

Loge

JEANNE : ... Le souffle du large...

Cabine

JEAN : ... L'ouragan des bravos...

Loge

JEANNE : ... Les nuits de quart...

Cabine

JEAN : ... Les soirs de gloire... (Gantillon 1928b : 97-98)

Cette alternance de phrases exprime sans aucun doute la tristesse et c'est ainsi que Gantillon construit un dialogue qui ne décrit pas, mais évoque les vives émotions des protagonistes comme le faisaient les Allemands : 
étant donné que le drame expressionniste n'utilise pas le dialogue à des fins d'analyse mais démontre, scéniquement, une construction de pièces qui procède par une succession rapide de scènes. Le visuel règne sur l'intellectuel. Même l'espace et le temps ne deviennent que de simples organes fonctionnels. Ou bien le temps est accéléré, ou bien son déroulement est inversé. Les effets optiques de la représentation suppléent à l'analyse. Lémotion se manifeste en tant qu'action. La diction expressionniste, elle non plus, ne décrit pas, elle ne s'adapte pas aux différents personnages selon des modes différents ; il faut, de même, la considérer beaucoup plus comme un instrument fonctionnel. Son changement est uniquement conditionné par une modification des états d'âme (Steffens 1993 : 162).

\section{En guise de conclusion}

Malgré certaines faiblesses, qui ne semblent pas aujourd'hui discutables, les drames de Gantillon paraissent particulièrement novateurs au public français des années vingt et trente du siècle écoulé. La recette de son succès réside dans le fait qu'il est inscrit parmi les artistes se moquant du réalisme et du rationalisme. Sa célébrité est due aussi à la création de protagonistes harcelés par leurs instincts contradictoires face à la nature à la fois menaçante et révélatrice à leur égard :

[...] trois spectacles d’ordre poétique où la Mer est, à la fois substance et force d'inspiration. Sa face féerique, elle apparaît derrière les visages des héros ; sa rumeur gronde à travers leurs voix, dans leur sang bat son pouls tumultueux.

Cyclone, Maya, Départs forment donc, non seulement une suite de portraits et de paysages marins, mais une trilogie d'identique résonance, chantant avec amour fraternel la geste des gens. L'auteur y a tenté de rendre sensible à l'esprit, sous les enluminures dramatiques, l'éternel conflit du rêve et de la vie; il a voulu surtout célébrer à son tour l'indétrônable primauté de l'imaginaire sur le réel (Gantillon 1928a : 11).

La conception du personnage de Gantillon se rapproche sans aucun doute de celle de la tradition expressionniste. Là-dessus, il faut souligner la psychologie des protagonistes qui n'a rien à voir avec les habitudes du théâtre réaliste, car ce théâtre rejette résolument l'unité du moi, qui ne pourrait être, comme l'écrit Théodule Ribot, que l'invention des spiritualistes. Le moi, selon le philosophe français, est, comme le cite Guy Vogelweith, « la coordination d'un certain nombre d'états sans cesse renaissant, ayant pour seul point d'appui le sentiment vague de notre corps » (Vogelweith 1971 : 112). Cette unité du moi n'est qu'une construction de l'intellect humain qui essaie de figer ce qui est continuellement changeable. Cette conception du caractère s'avère défaillante puisqu'elle suppose que l'homme est toujours identique et immuable, indépendamment des circonstances. Gantillon préfère, en revanche, les personnages intérieurement déchirés dont le comportement est souvent imprévisible. Puisant dans les profondeurs de l'âme humaine, le dramaturge découvre qu'à part ce moi qui pense, il y a encore des couches psychiques qui appartiennent à son être, mais qu'il ignore absolument. Nous n'avons donc pas 
là de caractères moliéresques qui se découvrent devant nous, encore qu'ils parlent comme de vrais êtres humains. Le dramaturge entend examiner le conflit interne de l'âme de l'homme qui séparpille en divers éléments discordants. Il étale des protagonistes dont l'identité se dérobe à la raison ; l'individu se présente en mouvement perpétuel et la connaissance que nous pouvons en avoir est toujours incertaine. Alors, un tel être, à qui l'on ne peut attribuer des traits de caractères fixes, s'avère être plus proche de la poétique expressionniste que des concepts cartésiens auxquels rend hommage le théâtre français.

\section{Références bibliographiques}

Bablet, D. (1971). L'Expressionnisme. In L’Expressionnisme dans le théâtre européen. Paris : Éditions du CNRS.

Gantillon, S. (1923). Cyclone. Collection nouvelle de la France dramatique. Paris : Librairie Stock.

- (1827). Maya. Masques, cinquième cahier.

—. (1928a). Marines. Paris : Société générale d'imprimerie et d'édition.

- (1928b). Départs. Masques, quinzième cahier.

Gravier, M. (1971). L'expressionnisme dramatique en France entre les deux guerres. In L'expressionnisme dans le théâtre européen (dir. D. Bablet, J. Jacquot). Paris : Éditions du CNRS.

Jomaron, J. de. (1981). La mise en scène contemporaine, II, 1914-1940. Bruxelles : La Renaissance du livre.

Raymond, M. (1943). Le Jeu retrouvé. Montréal : Éditions de l'Arbre.

Sarrazac, J.-P. \& Marcerou, Ph. (dir.) (1999). Antoine, linvention de la mise en scène, anthologie des textes d'André Antoine. Paris : Centre National du Théâtre. Actes Sud-Papiers.

Schopenhauer, A. (1970). Le Monde comme volonté et représentation. Paris : PUF.

Simon, A. (1970). Dictionnaire du théâtre français contemporain. Paris : Larousse.

Steffens, W. (1993). Théâtre. In Encyclopédie de l'expressionnisme (dir. L. Richard). Paris : Somogy.

Strindberg, A. (2006). Le Songe. Besançon : Éditions Les Solitaires Intempestifs.

Surer, P. (1964). Le Théâtre français contemporain. Paris : Société d'Édition et d'Enseignement Supérieur.

Tariol, M. (1980). Gaston Baty et Racine. In Hommage à Marcel Tariol. Toulouse : L'Université de ToulouseLe Miral.

Villeroy, A. (1923). Bulletin de la Chimère,(vol. 13).

Vogelweith, G. (1971). Le personnage et ses métamorphoses dans le théâtre de Strindberg. Lille : Service de reproduction des thèses de l'Université de Lille III . 